\title{
Neo Fuzzy Neuron Filters and Their Applications to Image Signal Preprocessing
}

\author{
Student Member Noriaki Suetake \\ Non-member \\ Takeshi Yamakawa \\ (Kyushu Institute of Technology) \\ (Kyushu Institute of Technology)
}

We propose novel nonlinear filters which are the extensions of a linear FIR filter and an order statistic (OS) filter by employing the neo fuzzy neuron (NFN) model. We also propose the hybrid type nonlinear filter aiming at elimination of a Gaussian noise and an impulsive noise at the same time, and high restoration of the signal, simultaneously. The proposed filters are synthesized by a learning method which guarantees optimal design caused by employing the NFN model. Moreover, the filters are effective not only for noise elimination but also for sharpening, edge extraction, and other various applications, because their functions are determined by the pairs of target and input signals in the training. The effectiveness and validity of the proposed filters are verified by applying them to the preprocessing of the image signals.

Keywords: Neo fuzzy neuron, Nonlinear filter, Linear FIR filter, Order statistic filter, Noise elimination, Sharpening, Edge extraction, Signal restoration, Image signal preprocessing

\section{Introduction}

A median filter is a representative nonlinear filter, and it offers excellent performance. It preserves edges or monotonic changes in trend, while eliminating impulses ${ }^{(1)}$. This performance can't be achieved by linear filters. However, a median filter is inferior to a linear filter with respect to elimination of a non-impulsive noise such as a Gaussian noise ${ }^{(2)}$. In order to inherit the advantages from both of a median filter and a linear filter, some filters such as the $\alpha$-trimmed mean filter have been developed ${ }^{(3)-(6)}$.

These filters based on a median filter, which is designed to eliminate the noise, are not always effective for the restoration of nonstationary signals such as image signals, because a median filter has disadvantage with respect to the preservation of the signals ${ }^{(7)}$. By sorting signal levels in the filter window, a median filter loses information of signal patterns. On the other hand, although a mean filter is a linear FIR filter which keeps pattern information in the filter window, it can't preserve the patterns such as edges of patterns well, because it has equal filter coefficients and is a lowpass filter which smoothes the signals. In short, for a restoration of the signal, the signal pattern in the window should be reflected to the design of the filter. Filter coefficients should be assigned in accordance with each point in the filter window and optimized ${ }^{(8)}$. From these view points, there are some filters designed using neural networks. However these algorithms have a disadvantage of local minimum problem ${ }^{(9)}$.

In this paper, we propose new nonlinear filters employing neo fuzzy neuron (NFN) model. The NFN model is presented by one of the authors ${ }^{(10)-(12)}$. The nonlinear characteristics of the NFN model is represented by fuzzy if-then rules with complementary mem- bership functions. A multi-input one-output NFN model, but not a neural network, can exhibit its good ability to describe a nonlinear relationship between inputs and output as well as its short learning time in comparison with a conventional multilayerd neural network. Furthermore, the NFN model guarantees a convergence to the global minimum ${ }^{(11)}$. The novel filters employing this NFN model include the following three types of filters:

1 FIR type NFN filter,

2 Order statistic (OS) type NFN filter,

3 FIR-OS hybrid type NFN filter.

The FIR type NFN filter, which aims particularly at restoration of the signal, is based on a framework of a linear FIR filter and the NFN model. In this filter, weights of the network are assigned in accordance with each point in the filter window and optimized. The OS type NFN filter, which aims particularly at elimination of noises, is based on a framework of an order statistic filter and the NFN model. An OS filter is generalization of a median filter and effective for the noise elimination. The hybrid type NFN filter achieves both of high noise elimination and high restoration simultaneously by reflecting both of the pattern information and statistical information in the window to its function ${ }^{(13)-(16)}$. Three types of filters above are synthesized by the learning which guarantees optimal design caused by employing the NFN model. And the filters are effective not only for noise elimination but also for sharpening, edge extraction, and so on, because their functions are determined by the pairs of target and input signals in the training. The effectiveness and the validity of the proposed filters have been verified by applying them to the preprocessing of the image signal. 


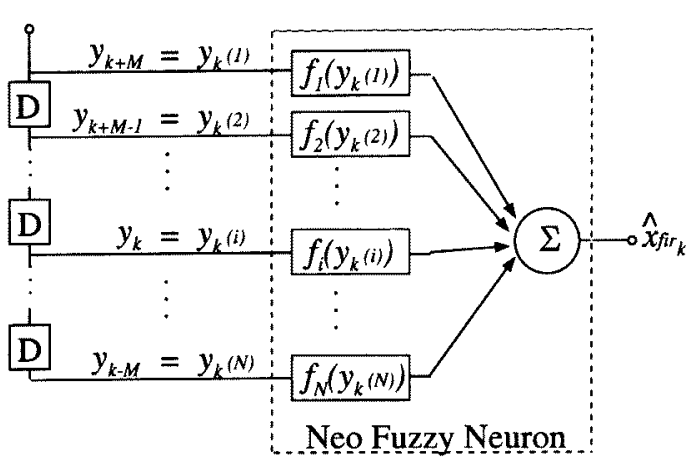

(a) (b)

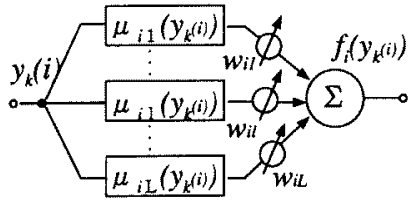

(c)

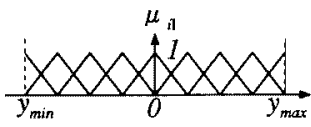

Fig. 1. Structures of the FIR type NFN filter and NFN model. (a) Structure of the FIR type NFN filter. (b) Structure of the nonlinear synapse which is described with a set of fuzzy if-then rules including singletons in consequents. (c) Triangular and complementary membership functions assigned for the fuzzy segments in the input space.

\section{Neo fuzzy neuron filters}

In this section, the NFN filters are discussed. Let's consider the following observation mechanism:

$$
y_{k}=x_{k}+v_{k},
$$

where $y_{k}$ is a noisy observation, $x_{k}$ an original signal, and $v_{k}$ an observation noise of an arbitrary distribution type.

2.1 FIR type NFN filter The output of a linear FIR filter of length $N$ operating on a sequence $\left\{y_{k}\right\}$ for $N$ odd is given by:

$$
\hat{x}_{k}=\sum_{i=1}^{N} \alpha(i) y_{k}(i),
$$

where $(\cdot)$ represents time/space sequence in the filter window. $y_{k}(1), \cdots, y_{k}(N)$ correspond to $y_{k-M}, \cdots, y_{k+M}$ respectively, and $M=(N-1) / 2$. The $\alpha(i)$ are constants that should be assigned for the sequential data in the filter window.

The proposed FIR type NFN filter employs a framework of a linear FIR filter and the NFN model. Fig.1 shows the structures of the proposed FIR type NFN filter and the NFN model. In this proposed filter, the estimation of an original signal $\hat{x}_{f i r_{k}}$ is given by:

$$
\hat{x}_{f i r_{k}}=\sum_{i=1}^{N} f_{i}\left(y_{k}(i)\right)
$$

with

$$
f_{i}\left(y_{k}(i)\right)=\frac{\sum_{l=1}^{L} \mu_{i l}\left(y_{k}(i)\right) w_{i l}}{\sum_{l=1}^{L} \mu_{i l}\left(y_{k}(i)\right)} .
$$

In Eq.(4), $l$ is the number assigned to labels of fuzzy segments, and $\mu_{i l}(\cdot)$ are membership functions. $w_{i l}$ are weights of the network. An input signal $y_{k}(i)$ activates only two membership functions simultaneously and the sum of grades of these two neighboring membership functions labeled by $l$ and $l+1$ is always equal to 1 , that is $\mu_{i l}\left(y_{k}(i)\right)+\mu_{i l+1}\left(y_{k}(i)\right)=1$, so that the defuzzification taking a center of gravity doesn't need a division and the output of the nonlinear synapse $f_{i}\left(y_{k}(i)\right)$ is rewritten from Eq.(4) as follows:

$$
f_{i}\left(y_{k}(i)\right)=\sum_{l=1}^{L} \mu_{i l}\left(y_{k}(i)\right) w_{i l} .
$$

The learning of the weights $w_{i l}$ is achieved so that the following error function $E_{f i r}\left(\hat{x}_{f i r_{k}}, t_{k}\right)$ becomes minimum:

$$
\begin{aligned}
& E_{f i r}\left(\hat{x}_{f i r_{k}}, t_{k}\right) \\
& \quad=\frac{1}{2} \sum_{k=1}^{K}\left(\hat{x}_{f i r_{k}}-t_{k}\right)^{2} \\
& \quad=\frac{1}{2} \sum_{k=1}^{K} \sum_{i=1}^{N} \sum_{l=1}^{L}\left(u_{i l}\left(y_{k}(i)\right) \cdot w_{i l}-t_{k}\right)^{2},
\end{aligned}
$$

where $t_{k}$ is a target signal and $K$ is the length of target signal. The gradient descent method is employed here, rather than least mean square (LMS) algorithm, since the former is easier to handle and simpler to calculate, especially in case of a large amount of weights. The learning is achieved for the pairs of training series of $y_{k}(i)$ and $t_{k}$ by using following equation:

$$
w_{i l}^{\text {new }}=w_{i l}^{\text {old }}-\eta_{f i r} \frac{\partial E_{f i r}\left(\hat{x}_{f i r_{k}}, t_{k}\right)}{\partial w_{i l}^{\text {old }}},
$$

where $\eta_{f i r}$ is the learning rate. Eq.(6) is unimodal function, because it is parabolic with respect to weights $w_{i l}$, that is, this NFN model guarantees the global minimum.

After the learning is completed, the set of weights of the network is optimized where the filtering output is as close to the ideal signal as possible.

2.2 OS type NFN filter The output of an OS filter of length $N$ operating on a sequence $\left\{y_{k}\right\}$ for $N$ odd is given by:

$$
\hat{x}_{k}=O S F\left(\left\{y_{j}\right\}_{j=k-M}^{k+M}\right)=\sum_{i=1}^{N} \alpha[i] y_{k}[i],
$$

where $y_{k}[1], \cdots, y_{k}[N]$ are the order statistics of $y_{k-M}, \cdots, y_{k+M}$, respectively. [.] represents order 


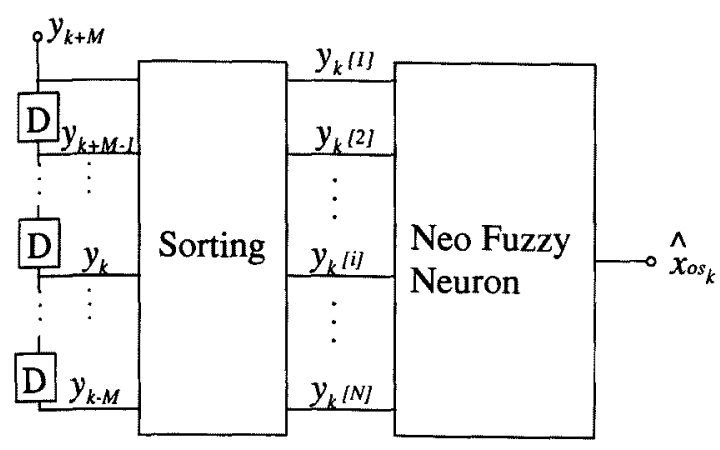

Fig. 2. Structure of the OS type NFN filter.

statistic of the signal. The $\alpha[i]$ are constants that is chosen for every application. An OS filter is a generalization of a median filter, a maximum filter and so on $^{(17)-(18)}$. For example, a median filter is a particular case of Eq.(8) with coefficients

$$
\alpha[i]= \begin{cases}1 & i=(N+1) / 2 \\ 0 & \text { otherwise }\end{cases}
$$

We can also define a maximum filter, for example, by taking

$$
\alpha[i]= \begin{cases}0 & i=1, \cdots, N-1, \\ 1 & i=N .\end{cases}
$$

The proposed OS type NFN filter employs a framework of an OS filter and the NFN model. Fig. 2 shows the structure of the proposed OS type NFN filter. In the proposed filter, the estimation of an original signal $\hat{x}_{o s_{k}}$ is given by:

$$
\hat{x}_{o s_{k}}=\sum_{i=1}^{N} f_{i}\left(y_{k}[i]\right)
$$

with

$$
f_{i}\left(y_{k}[i]\right)=\sum_{l=1}^{L} \mu_{i l}\left(y_{k}[i]\right) w_{i l} .
$$

The learning of the weights $w_{i l}$ is achieved so that the following error function $E_{o s}\left(\hat{x}_{o s_{k}}, t_{k}\right)$ becomes minimum:

$$
E_{o s}\left(\hat{x}_{o s_{k}}, t_{k}\right)=\frac{1}{2} \sum_{k=1}^{K}\left(\hat{x}_{o s_{k}}-t_{k}\right)^{2} .
$$

The gradient descent method is employed here. The learning is achieved for the pairs of training series of $y_{k}[i]$ and $t_{k}$ by using following equation:

$$
w_{i l}^{n e w}=w_{i l}^{o l d}-\eta_{o s} \frac{\partial E_{o s}\left(\hat{x}_{o s_{k}}, t_{k}\right)}{\partial w_{i l}^{\text {old }}},
$$

where $\eta_{o s}$ is the learning rate. It is also easily understood as discussed above that the global minimum is reached.

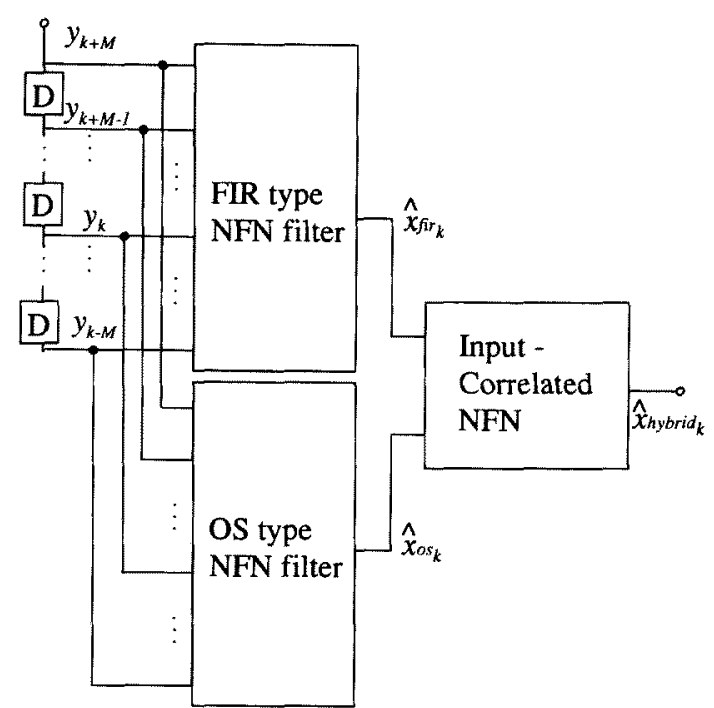

Fig. 3. Structure of the FIR-OS hybrid type NFN filter.

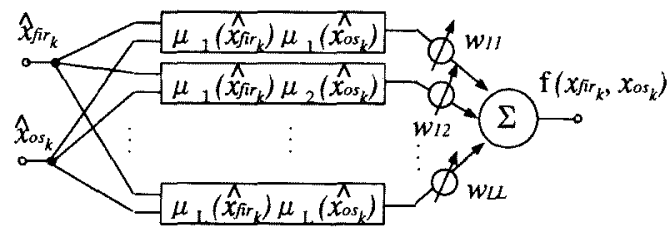

Fig. 4. Structure of an input-correlated nonlinear synapse. This synapse acts as a two-input one-output fuzzy inference engine input output relation of which is assigned by the learning.

2.3 FIR-OS hybrid type NFN filter The proposed FIR-OS hybrid type NFN filter is shown in Fig.3, where the outputs from the FIR type and OS type NFN filters are fed to the Input-Correlated NFN. In this case, the output of the filter is obtained as an output of the input-correlated nonlinear synapse. Fig.4 shows the structure of the input-correlated nonlinear synapse. As shown in Fig.4, this nonlinear synapse acts as a fuzzy inference engine the input output relation of which is assigned by learning.

In the proposed filter, the estimation of an original signal $\hat{x}_{\text {hybrid }_{k}}$ is given by:

$$
\hat{x}_{\text {hybrid }_{k}}=f\left(\hat{x}_{f i r_{k}}, \hat{x}_{o s_{k}}\right)
$$

with

$$
f\left(\hat{x}_{f i r_{k}}, \hat{x}_{o s_{k}}\right)=\sum_{l=1}^{L} \sum_{l=1}^{L} \mu_{l}\left(\hat{x}_{f i r_{k}}\right) \mu_{l}\left(\hat{x}_{o s_{k}}\right) w_{l l} .
$$

where $\hat{x}_{f i r_{k}}$ and $\hat{x}_{o s_{k}}$ are given by Eqs.(3) and (11). The learning can be achieved by the following three stages.

1) Learning of the FIR type NFN filter 
All the weights in the FIR type NFN filter are assigned by applying input sequential data $y_{k-M}, \cdots, y_{k}, \cdots, y_{k+M}$ and target signal $t_{k}$ to minimize $E_{f i r}\left(\hat{x}_{f i r_{k}}, t_{k}\right)$, where the gradient descent method is employed.

\section{2) Learning of the OS type NFN filter}

All the weights in the OS type NFN filter are assigned by applying input sequential data $y_{k-M}, \cdots, y_{k}, \cdots, y_{k+M}$ and target signal $t_{k}$ to minimize $E_{o s}\left(\hat{x}_{o s_{k}}, t_{k}\right)$, where the gradient descent method is employed.

\section{3) Learning of the Input-Correlated NFN}

Outputs of FIR type and OS type NFN filters, with fixed weights after learning, are connected with two inputs of the Input-Correlated NFN. All the weights of the Input-Correlated NFN are assigned by employing input sequential data $y_{k-M}, \cdots, y_{k}, \cdots, y_{k+M}$ and target signal $t_{k}$ to minimize $E_{\text {hybrid }}\left(\hat{x}_{\text {hybrid }}, t_{k}\right)$ (Eq.(17)), where the gradient descent method is employed.

$$
E_{h y b r i d}\left(\hat{x}_{h y b r i d_{k}}, t_{k}\right)=\frac{1}{2} \sum_{k=1}^{K}\left(\hat{x}_{h y b r i d_{k}}-t_{k}\right)^{2} .
$$

The same target signal $t_{k}$ is employed for all learning processes of 1), 2) and 3). The learning of the InputCorrelated NFN is achieved by following equation:

$$
w_{l l}^{\text {new }}=w_{l l}^{\text {old }}-\eta_{h y b r i d} \frac{\partial E_{h y b r i d}\left(\hat{x}_{h y b r i d_{k}}, t_{k}\right)}{\partial w_{l l}^{\text {old }}},
$$

where $\eta_{h y b r i d}$ is the learning rate. It is also easily understood that the global minimum is reached.

\section{Experimental results}

The attempt is made to verify the effectiveness and the validity of the proposed filters by applying them to the preprocessing of the image signal. In the experiments, the signals in the filter window are numbered and fed to a filter as shown in Fig.5. Here, the filter window size $N$ is $9(=3 \times 3$ pixels) and the number of labels of fuzzy segments in brightness (level) of each pixel is 15 . Learning rates $\eta_{f i r}, \eta_{o s}$ and $\eta_{\text {hybrid }}$ are assigned

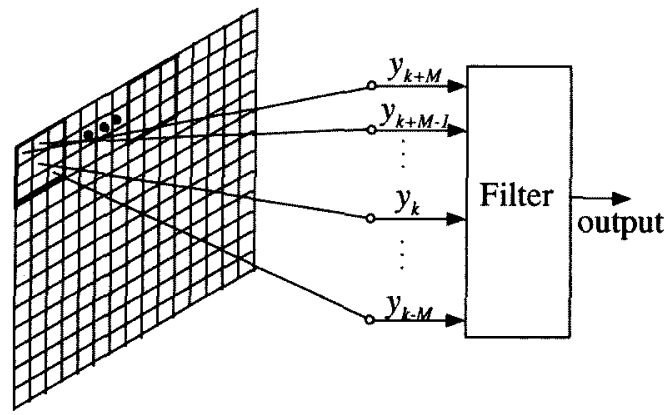

Fig. 5. How to input the image data to the filters. to be 0.01 in each learning process of the FIR type, the OS type and the FIR-OS hybrid type NFN filter.

3.1 Noise elimination Here, the proposed filters are applied to the noise elimination for the images of machine printed capital characters and human faces.

\subsubsection{Machine printed capital characters}

Learning process In this experiment, we employed the images shown in Fig.6(a) and (b), which are constructed with $100 \times 100$ pixels and the resolution is 8 bits/pixel gray-level, as a common target image and the input image for each learning process of the FIR type, the OS type and the FIR-OS type NFN filter, respectively. In order to clarify the effects of filtering, bird's-eye view images are employed here. The input image shown in Fig.7(b) is the target image corrupted by both of a Gaussian noise $\mathrm{N}(0,200)$ and an impulsive noise $(2 \%)$, the elimination of which is very difficult for the conventional filters ${ }^{(19),(20)}$. Each learning of the FIR type, the OS type and the FIR-OS hybrid type NFN filter was completed after about 400 iterations (one iteration is defined as renewals for all pixels in one training image.)

Testing process After each learning has been completed, the performances of the proposed filters are tested for the images of machine printed capital characters ( $A$ to $\mathrm{Z}$ ). For examples, we show the results of noise elimination for the images of machine printed capital character ' $G$ ' $(50 \times 50$ pixels, 8 bits/pixel gray-level). Fig.7(a) shows an original image ' $G$ '. Fig.7(b) shows the input image which is the original image corrupted by both of a Gaussian noise $\mathrm{N}(0,200)$ and an impulsive

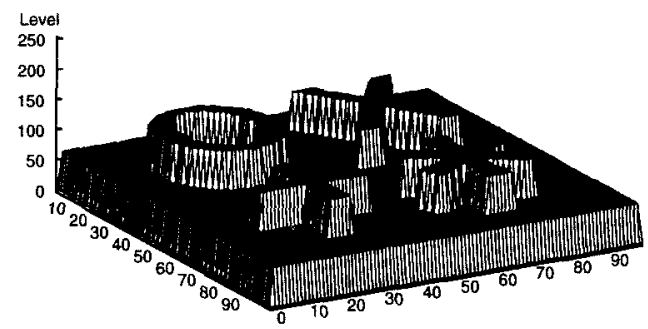

(a)

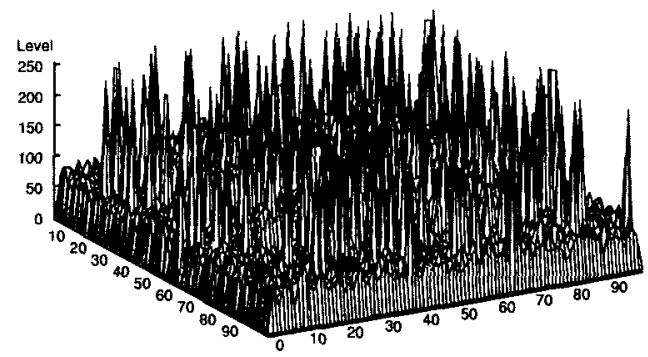

(b)

Fig. 6. Training data for the machine printed capital characters. (a) A target image in the learning (b) An input image, which is the target image corrupted by a Gaussian noise $\mathrm{N}(0,200)$ and an impulsive noise $(2 \%)$, in the learning. 


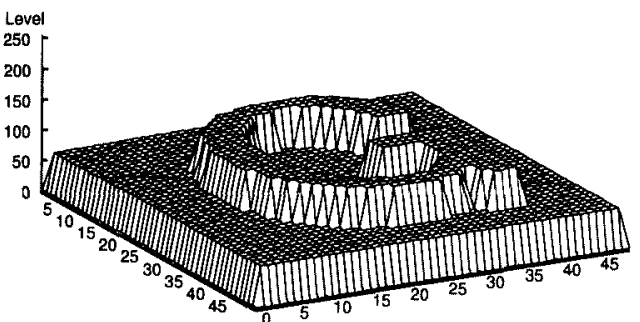

(a)

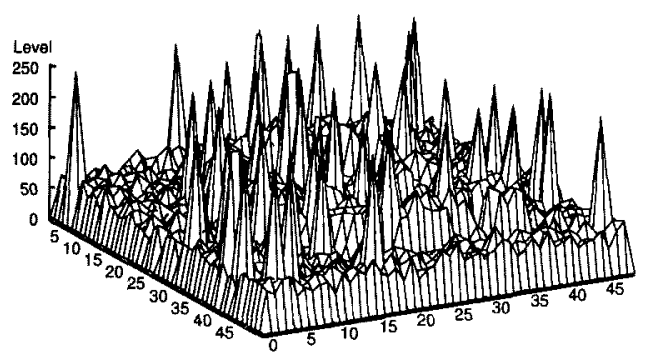

(b)

Fig. 7. Testing data for the machine printed capital character ' $G$ '. (a) An original image in the filtering. (b) An input image, which is the original image corrupted by a Gaussian noise $\mathrm{N}(0,200)$ and an impulsive noise ( $2 \%$ ), in the filtering.

noise (2\%), similar to Fig.6(b). The root mean square error (RMSE) of the input image shown in Fig.7(b) is 28.8. The RMSE is calculated by the following equation:

$$
R M S E=\frac{1}{P \times Q} \sqrt{\sum_{i=1}^{P} \sum_{j=1}^{Q}(y(i, j)-x(i, j))^{2}}
$$

where $P \times Q$ is an image size, $y(i, j)$ the input image and $x(i, j)$ the original image. Fig.8(a), (b) and (c) show the results of filtering by the FIR type, the OS type, and the FIR-OS hybrid type NFN filter, respectively. The RMSE of the FIR type, the OS type and the FIR-OS hybrid type NFN filter are 7.9, 6.4 and 4.4, respectively. For comparison, an optimized linear FIR filter and an optimized OS filter are examined and the results of filtering by them are shown in Fig.8(d) and (e), respectively. The RMSE of an optimized linear FIR and an OS filter are 13.7 and 8.4, respectively.

In the images, the levels of the parts of the character and the background are 128 and 64 , respectively. Fig. 9 shows the results of filtering by the proposed filters, which are ordinary images not bird's-eye view images. From these results, it seems reasonable to say that the FIR type NFN filter keeps the patterns of edge, the OS type NFN filter keeps patterns of the character and the background parts, and the FIR-OS hybrid type NFN filter keeps the both patterns of edges, and character and background parts well. In other words, the FIR type NFN filter is effective for the restoration of the signal, the OS type NFN filter is effective for the elimination of noises, and the FIR-OS hybrid type NFN filter achieves

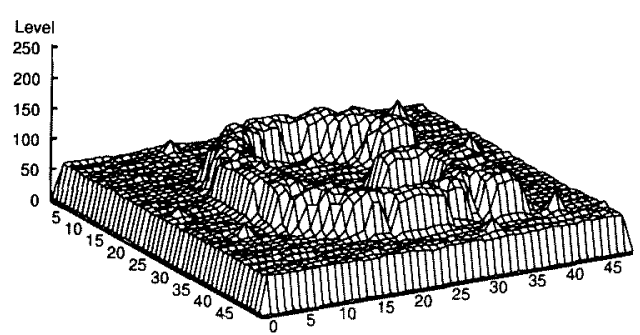

(a)

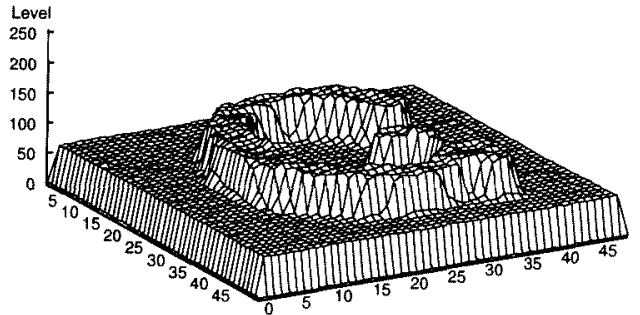

(b)

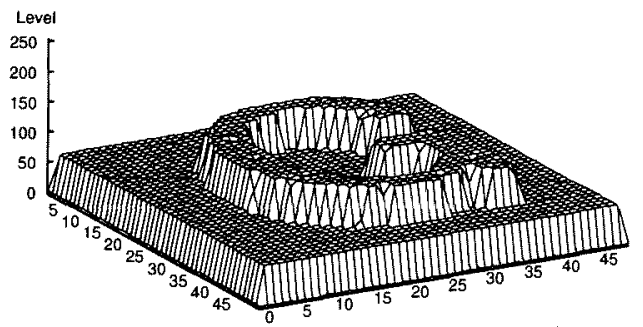

(c)

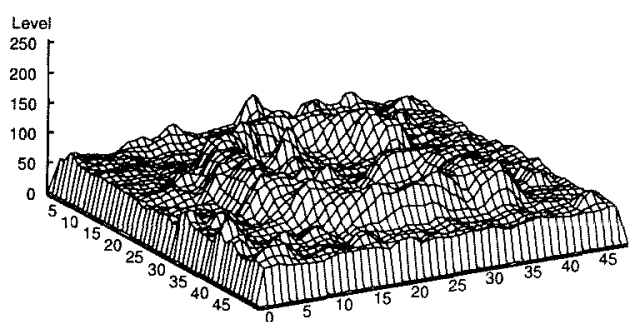

(d)

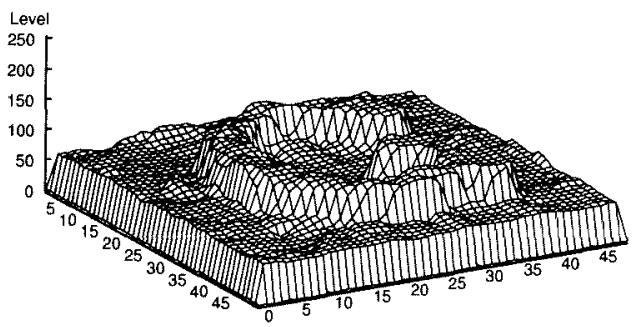

(e)

Fig. 8. The filtering results for the machine printed capital character ' $G$ '. (a), (b) and (c) show the results of filtering by the FIR type, the OS type, and the FIR-OS hybrid type NFN filter, respectively. (d) and (e) show the results of filtering by an optimized linear filter and an optimized OS filter, respectively.

both of high noise elimination and high restoration of 


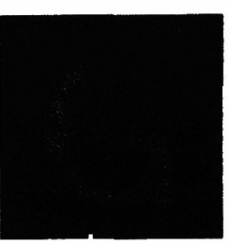

(a)

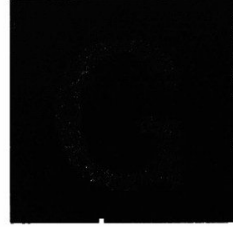

(b)

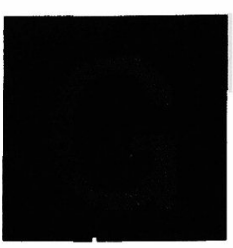

(c)
Fig. 9. The results of filtering by the proposed filters. (a), (b) and (c) show the results of filtering by the FIR type, the OS type and the FIR-OS hybrid type NFN filters, respectively.

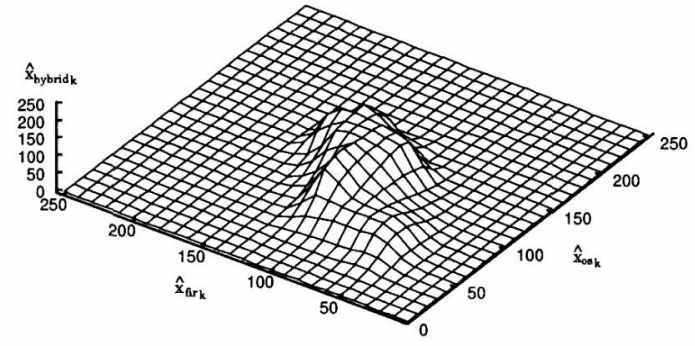

Fig. 10. The input-output characteristics of the input-correlated nonlinear synapse in the FIR-OS hybrid type NFN filter.

the signal, simultaneously.

In Fig.10, we show the input-output characteristics of the input-correlated nonlinear synapse in the FIR-OS hybrid type NFN filter shown in Fig.4. This characteristics can be represented by fuzzy if-then rules explicitly, because the input-correlated nonlinear synapse is equal to a fuzzy inference engine as mentioned above. From Fig.10, the following three fuzzy if-then rules are extracted roughly:

[Rule 1]

If $\hat{x}_{f_{i r_{k}}}$ is about 128 (level of character part) level and $\hat{x}_{o s_{k}}$ is about 60-150 level, then $\hat{x}_{\text {hybrid }_{k}}$ is about 128 level.

[Rule 2]

If $\hat{x}_{\text {fir }_{k}}$ is about 50-100 level and $\hat{x}_{o s_{k}}$ is about 64 (level of background part) level, then $\hat{x}_{\text {hybrid }_{k}}$ is about 64 level.

[Rule 3]

If $\hat{x}_{\text {fir }_{k}}$ is about 90-150 level and $\hat{x}_{o s_{k}}$ is about 128 level, then $\hat{x}_{\text {hybrid }_{k}}$ is about 128 level.

Rule 1 reflects the effect of the FIR type NFN filter mainly, because fuzziness of $\hat{x}_{f i r_{k}}$ is weaker than that of $\hat{x}_{o s_{k}}$ and the rule reacts sensitively to the value of $\hat{x}_{f i r_{k}}$ in contrast with that of $\hat{x}_{o s_{k}}$. As shown Fig.9(a), 128 level in the output of the FIR type NFN filter represents level of the character part including edges in the image. Rule 2 and 3 reflect the effect of the OS type NFN filter mainly, similar to Rule 1. As shown Fig.9(b), 64 and 128 levels in the output of the OS type NFN filter represent levels of the background part and inside region of the character part in the image, respectively.
Table 1. RMSE for the 26 capital characters, and its average, covariance, maximum and minimum. 'Input' represents the corrupted input images. 'Con.1 and 2' represent an optimized FIR filter and an optimized OS filter, respectively. 'NFN 1, 2 and 3' represent the FIR type NFN filter, the OS type NFN filter and the FIR-OS hybrid type NFN filter, respectively. 'Ave.' , 'Var.', 'Max' and 'Min' represent the average, variance, maximum and minimum of the RMSE, respectively.

\begin{tabular}{|c|c|c|c|c|c|c|}
\hline & Input & Con. 1 & Con. 2 & NFN 1 & NFN 2 & NFN 3 \\
A & 25.8 & 14.2 & 10.5 & 10.4 & 9.6 & 8.3 \\
B & 27.2 & 14.7 & 9.6 & 9.9 & 8.6 & 7.4 \\
C & 28.8 & 14.8 & 10.6 & 10.7 & 10.1 & 9.0 \\
D & 28.5 & 13.5 & 8.1 & 8.5 & 6.8 & 5.7 \\
E & 28.8 & 15.6 & 11.5 & 10.1 & 9.0 & 7.9 \\
F & 28.7 & 14.6 & 11.5 & 10.3 & 8.8 & 8.1 \\
G & 28.8 & 13.7 & 8.4 & 7.9 & 6.4 & 4.4 \\
H & 27.5 & 15.6 & 11.4 & 11.1 & 9.9 & 9.0 \\
I & 29.2 & 12.2 & 8.5 & 8.7 & 7.5 & 6.9 \\
J & 26.2 & 14.7 & 11.3 & 11.9 & 10.7 & 10.3 \\
K & 27.5 & 14.7 & 10.6 & 9.6 & 7.9 & 7.0 \\
L & 26.6 & 12.2 & 7.8 & 7.8 & 6.4 & 5.7 \\
M & 30.2 & 15.2 & 10.8 & 9.1 & 8.1 & 6.7 \\
N & 29.2 & 14.1 & 9.8 & 9.0 & 7.7 & 6.5 \\
O & 27.0 & 14.7 & 9.9 & 9.2 & 7.9 & 6.8 \\
P & 29.7 & 13.6 & 9.4 & 8.8 & 7.1 & 6.1 \\
Q & 28.1 & 15.4 & 11.4 & 11.6 & 10.6 & 9.4 \\
R & 27.2 & 14.3 & 9.5 & 8.9 & 7.6 & 6.4 \\
S & 30.9 & 15.1 & 10.9 & 11.0 & 10.0 & 9.1 \\
T & 28.8 & 12.5 & 8.2 & 8.1 & 6.7 & 5.9 \\
U & 29.1 & 15.4 & 11.4 & 10.8 & 9.4 & 8.7 \\
V & 29.7 & 13.9 & 9.4 & 9.0 & 8.3 & 6.9 \\
W & 28.5 & 16.0 & 12.1 & 10.5 & 10.5 & 8.8 \\
X & 28.2 & 14.5 & 11.0 & 9.5 & 8.8 & 7.5 \\
Y & 31.1 & 14.3 & 10.4 & 9.8 & 8.7 & 8.0 \\
Z & 31.2 & 15.2 & 11.3 & 10.9 & 10.1 & 9.0 \\
\hline Ave. & 28.5 & 14.4 & 10.2 & 9.7 & 8.5 & 7.5 \\
Var. & 1.9 & 0.9 & 1.5 & 1.2 & 1.7 & 1.9 \\
Max. & 31.2 & 16.0 & 12.1 & 11.9 & 10.7 & 10.3 \\
Min. & 25.8 & 12.2 & 7.8 & 7.8 & 6.4 & 4.4 \\
\hline & & & & & & \\
\hline
\end{tabular}

Thus, we can say that Rule 1 acts for the edge preservation as the FIR type NFN filter does, and Rule 2 and 3 act for the noise elimination as the OS type NFN filter does. And this set of the fuzzy if-then rules, i.e., Fig.10 shows that the FIR-OS hybrid type NFN filter inherits advantages from both of the FIR type and the OS type NFN filter well.

Table 1 shows the RMSE for the images of the 26 (A to $\mathrm{Z}$ ) capital characters, and the average, variance, maximum and minimum of the RMSE. As shown Table 1 , it is clear that the results of the proposed filters are superior to other two filters.

Furthermore, the RMSE versus the parameters of various noises are investigated, some of which are shown in Figs.11 and 12. The superiority of the proposed filter to other two filters is easily understood.

3.1.2 Human facial image In this experiment, we employed the images $(120 \times 160$ pixels, 8 bits/pixel gray-level) shown in Fig.13(a) and (b), as a common target image and the input image for each larning process of the FIR type, the OS type and the FIR-OS type NFN filter, respectively. The input image shown in Fig.13(b) is the target image corrupted by both of a Gaussian noise $\mathrm{N}(0,200)$ and an impulsive noise $(2 \%)$. 


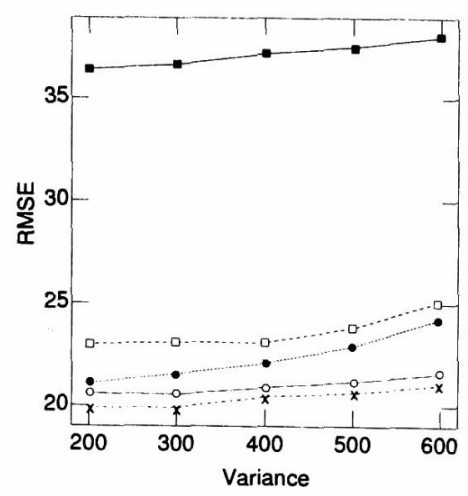

Fig. 11. The RMSE versus a variance of a Gaussian noise of a mixed noise. A percentage of an impulsive noise is fixed at $15 \%$. $\square$ and $\square$ represent RMSE of optimized linear FIR filter and order statistic filter, respectively. $\quad, O$ and $\times$ represent the RMSE of the FIR type, OS type, FIR-OS hybrid type NFN filter, respectively.

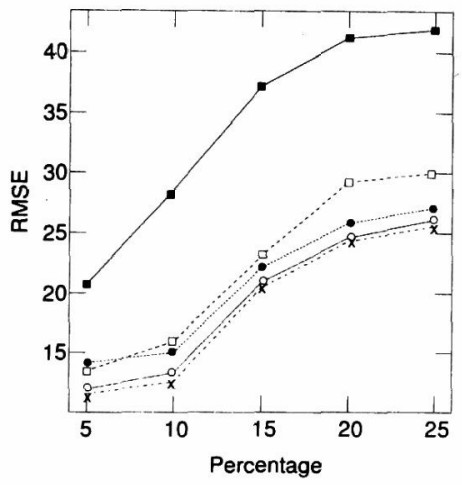

Fig. 12. The RMSE versus a percentage of an impulsive noise of a mixed noise. A variance of a Gaussian noise is fixed at 400 . The symbols represent RSME of the filters similar to Fig.11.

After each learning has been completed, the performances of the proposed filters are tested for the facial image. We show the results of noise elimination for the facial image. Fig.14(a) shows an original facial image $(120 \times 160,8$ bits/pixel gray-level). Fig.14(b) shows the input image which is the original image corrupted by both of a Gaussian noise $\mathrm{N}(0,200)$ and an impul-

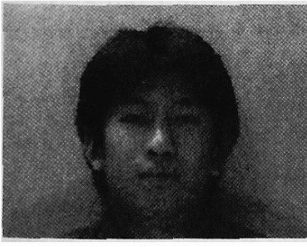

(a)

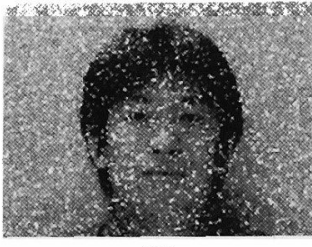

(b)
Fig. 13. Training data for the the facial images. (a) A target image in the learning. (b)An input facial image, which is the target image corrupted by both of a Gaussian noise $\mathrm{N}(0,200)$ and an impulsive noise $(2 \%)$, in the learning.

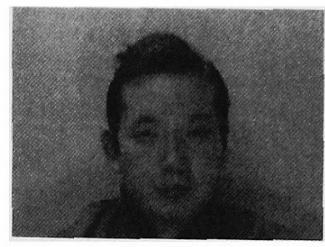

(a)

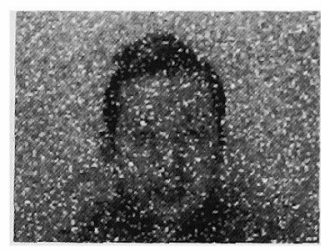

(b)
Fig. 14. Testing data for the facial images. (a)An original image in the filtering. (b)An input facial image, which is the original image corrupted by both of a Gaussian noise $\mathrm{N}(0,200)$ and an impulsive noise $(2 \%)$, in the filtering.

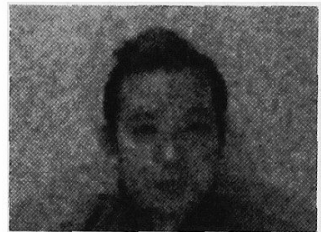

(a)

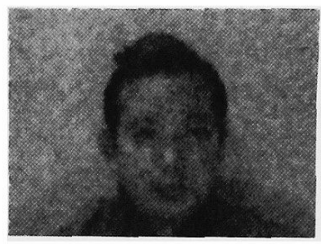

(c)

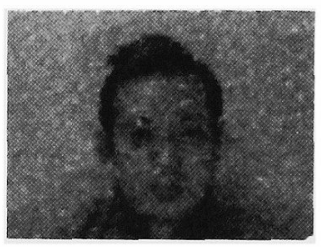

(e)

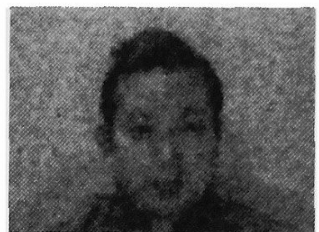

(b)

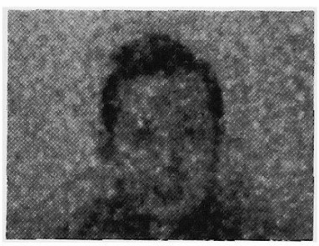

(d)
Fig. 15. The filtering results for the facial image. (a),(b) and (c) show the results of filtering by the FIR, OS, FIR-OS hybrid type NFN filter, respectively. (d) and (e) show the results of filtering by an optimized linear FIR filter and an optimized OS filter, respectively.

sive noise (2\%), similar to Fig.13(b). The RMSE of the input image shown in Fig.14(b) is 46.5. Fig.15(a),(b) and (c) show the results of filtering by the FIR type, the OS-type, and the FIR-OS hybrid type NFN filter respectively. The RMSE of the the FIR type, the OStype, and the FIR-OS hybrid type NFN filter are 9.7, 9.3 and 9.2 , respectively. For comparison, an optimized linear filter and an optimized OS filter are examined and the results of filtering by them are shown in Fig.15(d) and (e), respectively. The RMSE of the an optimized linear FIR filter and an optimized OS filter are 16.4 and 11.7 , respectively. The results of the proposed filters are superior to other two filters. 


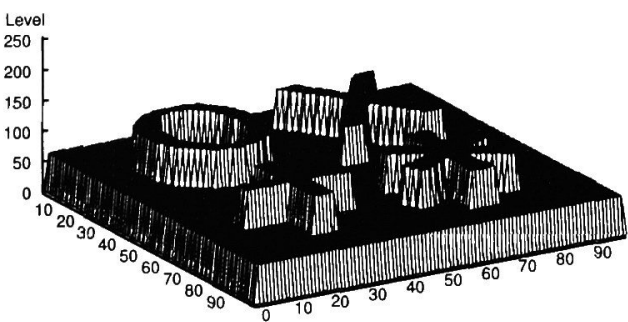

(a)

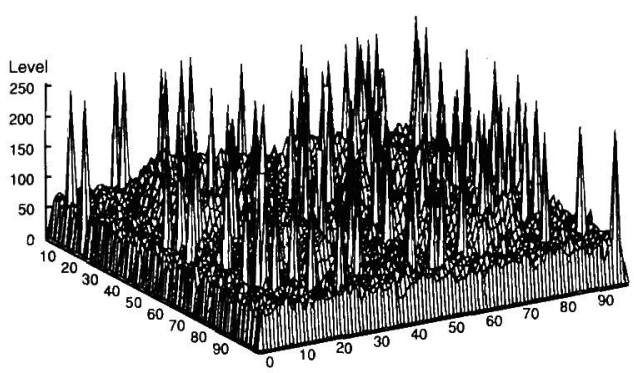

(b)

Fig. 16. Ttraining data for the sharpening. (a) A target image in the learning. (b) An input image, which is the target image smoothed by a mean filter and corrupted by both of a Gaussian noise $\mathrm{N}(0,100)$ and an impulsive noise (1\%), in the learning.

3.2 Sharpening of images The FIR-OS hybrid type filter is applied to the sharpening of the images of machine printed capital characters.

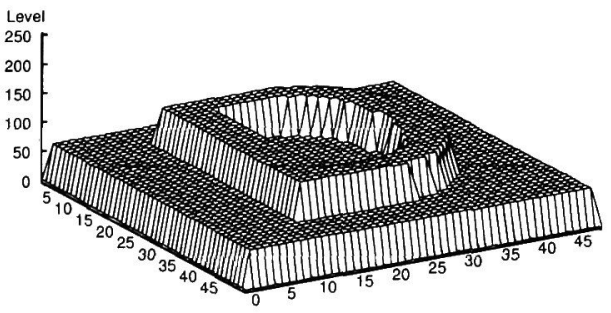

(a)

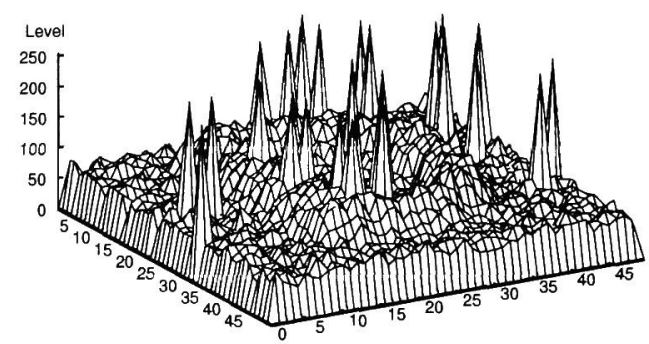

(b)

Fig. 17. Testing data for the sharpening. (a) An original image in the testing. (b) An input image, which is the original image smoothed by a mean filter and corrupted by both of a Gaussian noise $\mathrm{N}(0,100)$ and an impulsive noise $(1 \%)$, in the sharpening.

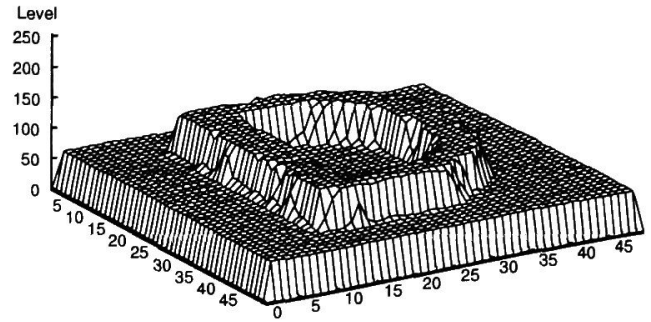

Fig. 18. The results of the sharpening by the FIR-OS hybrid type NFN filter.

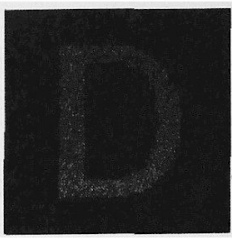

(a)

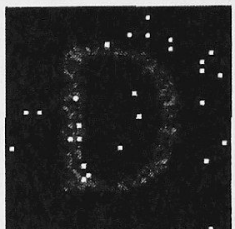

(c)

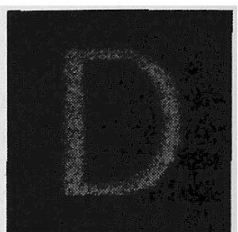

(b)

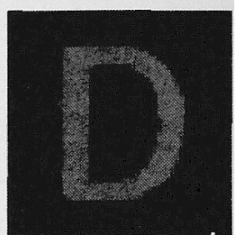

(d)
Fig. 19. The images employed in the sharpening. (a) An original image, (b)A smoothed original image by a mean filter, (c)An input image in the sharpening, (d)The results of the sharpening by the FIR-OS hybrid type NFN filter.

In this experiment, we employed the images shown Fig.16(a) and (b) $(100 \times 100$ pixels, 8 bits/pixel graylevel), as a target image and the input image in the learning process of the FIR-OS type NFN filter, respectively. The input image shown in Fig.16(b) is the target image corrupted by both of a Gaussian noise $\mathrm{N}(0,100)$ and an impulsive noise (1\%) after smoothing by a mean filter, widow size of which is $9(3 \times 3)$.

After the learning has been completed, the performances of the proposed filter are tested for the machine printed capital characters. For an example, the result of the sharpening for the capital character image ' $D$ ' is shown here. Fig.17(a) shows an original image $(50 \times 50$ pixels, 8bit/pixel gray-level). Fig.17(b) shows an original image and an input image which is the original image corrupted by both of a Gaussian noise $\mathrm{N}(0,100)$ and an impulsive noise $(1 \%)$ after smoothing it by a mean filter, window size of which is $9(3 \times 3)$ pixels, similar to Fig.16(b). The result of sharpening by the FIR-OS hybrid type NFN filter is shown in Fig.18. Furthermore, in order to clarify the effect of the proposed filter, we show the images employed in this experiment (sharpening), which are ordinary images not bird's-eye view images (Fig.19). From Figs.18 and 19, it is clear that the sharpening of the image is achieved well. The conventional 


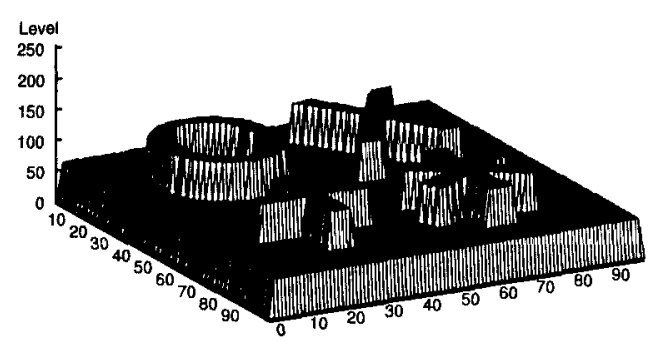

(a)

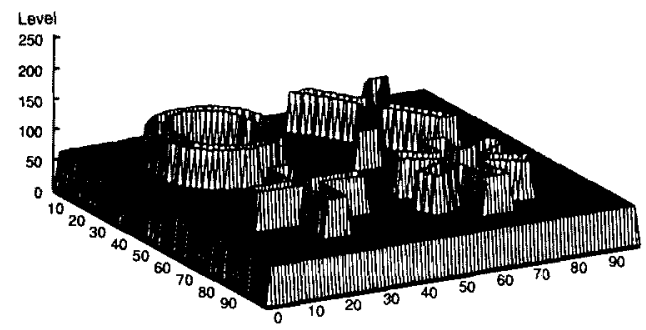

(b)

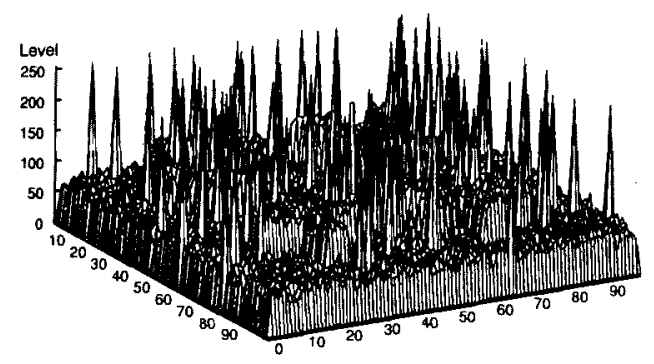

(c)

Fig. 20. Training data for the edge extraction. (a) An original image in the learning. (b) A target image in the learning. (c) An input image, which is the original image corrupted by both of a Gaussian noise $N(0,100)$ and an impulsive noise $(1 \%)$, in the learning.

filters are much less effective than the FIR-OS hybrid type NFN filter, because they don't have high mapping ability between input and output, like the NFN model.

3.3 Edge extraction The FIR-OS hybrid type filter is applied to the edge extraction of the images of machine printed capital characters.

In this experiment, we employed the images shown Fig.20(a), (b) and (c) $(100 \times 100$ pixels, 8 bits/pixel gray-level), as an original image, a target image and the input image in the learning process of the FIR-OS type NFN filter, respectively. The target image shown in Fig.20(b) is the image of the edges extracted from the original image. The input image shown in Fig.20(c) is the original image corrupted by both of a Gaussian noise $\mathrm{N}(0,100)$ and an impulsive noise $(1 \%)$, the edge extraction of which is very difficult for the conventional algorithms, such as Robert, Prewitt and Sobel operators based on the gradient of the image ${ }^{(2)}$. This difficulty is derived from that the both of a noise and edges have great gradient magnitude. In this case, smooth-

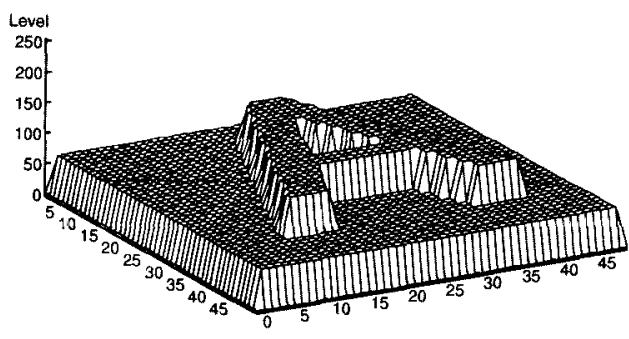

(a)

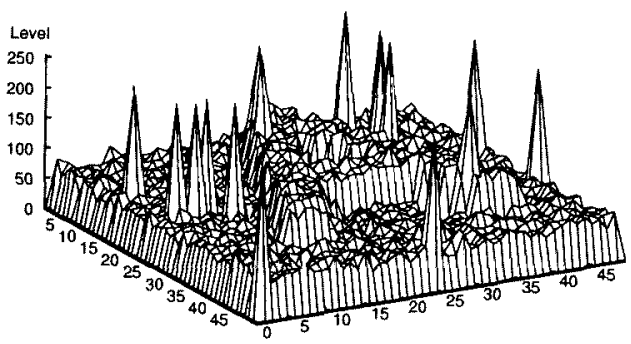

(b)

Fig. 21. Testing data for the edge extraction. (a) An original image in the testing. (b) An input image, which is the original image corrupted by both of a Gaussian noise $\mathrm{N}(0,100)$ and an impulsive noise $(1 \%)$, in the edge extraction.

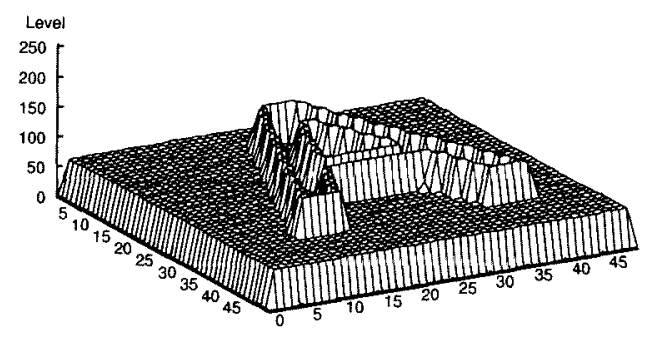

Fig. 22. The results of the edge extraction by the FIR-OS hybrid type NFN filter.

ing filters are commonly used as prefilters, however, it tends to reduce edge gradients, so that the correct edge extraction can not be often achieved.

After the learning has been completed, the performance of the proposed filter is tested for the machine printed capital characters. For an example, the result of the edge extraction for the capital character image 'A' is shown here. Fig.21(a) shows an original image $(50 \times 50$ pixels, 8 bit/pixel gray-level). Fig.21(b) shows an input image which is the original image corrupted by both of a Gaussian noise $\mathrm{N}(0,100)$ and an impulsive noise $(1 \%)$, similar to Fig.20(c). The result of the edge extraction by the FIR-OS hybrid type NFN filter is shown in Fig.22. The edge extraction of the image is accomplished enough.

\section{Conclusions}

In this paper, novel nonlinear filters which are extensions of a linear FIR filter and an OS filter by employing 
the neo fuzzy neuron (NFN) model, and their hybrid type nonlinear filter, were proposed. The filters are optimally designed and implemented by learning which guarantees convergence to the global minimum.

Through the experiments, we confirmed that the FIR type NFN filter is effective for the restoration of the signal, the OS type NFN filter is effective for the elimination of noises, and the FIR-OS hybrid type NFN filter significantly achieves both of high noise elimination and high restoration of the signal, simultaneously.

One of main characteristics of proposed filters is that they are applicable to arbitrary image signal preprocessing. Many of traditional filters are confined to specific use. On the other hand, our filters proposed here are effective not only for noise elimination but also for sharpening, edge extraction, and other various applicatons. These features of proposed filers are derived from that their functions are determined by the pairs of target and input signals in the training. If we prepare a typical training set of images for some practical purposes, we can tune the filters to be suitable for these purposes.

Furthermore, the proposed filters do not require a complicated algorithms, and their architectures are very simple. They have highly potential applications to a wide range of practical signal processing.

(Manuscript received February 23, 1999, revised May 17, 1999)

\section{References}

(1) J. W. Tukey: Nonlinear (nonsuperposable) Methods for Smoothing Data (1974) Congr. Rec. 1974 EASCON, 673

(2) Edited by IEICE: Digital Signal Processing Handbook(in Japanese) (1993) Ohm Inc.

(3) I. Pitas \& A. N. Venetsanopoulos: Nonlinear Mean Filters in Image Processing 1986 IEEE Trans. Acoust. Speech, and Signal Process. ASSP-34 (3) 573-584

(4) D. R. K. Brownringg: The Weighted Median Filter (1984) Communications of the ACM 27 (8) 807-818

(5) A. Asano, K. Itoh \& Y. Ichioka: Optimization of the Weighted Median filter by learning (1991) OPTICT LETTERS 16 (3) 168-170

(6) Y. Hoon \& A. T. Fam: An Edge Gradient Enhancing Adaptive Order Statistic Filter (1987) IEEE Trans. Acoust. Speech, and Signal Process. ASSP-35 (5) 680-695

(7) K. Arakawa \& H. Harashima: Intelligent Signal Processing:Its Present and Future(in Japanese) (1995) Trans. on IEICE. J78A (2) 103-113

(8) P. Heinonen \& Y. Neuvo: FIR-Median Hybrid Filters (1987) IEEE Trans. Acoust. Speech, and Signal Process. ASSP-35 (6) 832-838

(9) K. Arakawa, K. Yamakawa \& M. Koyama: Neural NetType Filter with Optimization of Its Nonlinear Function(in Japanese) (1995) Trans. on IEICE. J78-A (2) 151-160

(10) T. Yamakawa, E. Uchino, T. Miki \& H. Kusanagi: A Neo Fuzzy Neuron and Its Applications to System Identification and Prediction of the System Behavior (1992) Proc. of the 2nd Int. Conf. on Fuzzy Logic \& Neural Networks 477-483

(11) T. Yamakawa, H. Kusanagi, E. Uchino \& T. Miki: A New Effective Learning Algorithm for a Neo Fuzzy Neuron Model (1993) Proc. of the Fifth IFSA World Congress 1017-1020

(12) T. Yamakawa: Japanese Patent Application (1992) TOKUGAN-HEI 04-132897

(13) L. Yin, J. Astola \& Yrjo Neuvo: A New Class of Nonlinear Filters - Neural Filters (1993) IEEE Trans. Signal Process.
SP-41 (3) 1201-1222

(14) M. Meguro \& A. Taguchi: Learning Type of Median and Mean Hybrid Filters and a Synthesis of It's Learning Signal(in Japanese) (1998) Trans. on IEICE. J81-A (1) 44-55

(15) A. Taguchi \& M. Meguro: Adaptive Order Statistics Filters with High Robustness(in Japanese) (1997) Trans. on IEICE. J80-A (11) 2049-2053

(16) A. Taguchi, M. Muneyasu \& T Hinamoto: Median and Neural Networks Hybrid (MNNH) Filters (in Japanese) (1996) Trans. on IEICE. J79-A (11) 1817-1825

(17) K. Arakawa: Signal Processing and Nonlinearity(in Japanese) (1997) The Journal of IEICE. 80 (11) 1190-1194

(18) K. Arakawa: Nonlinear Digital Filters and Their Applications(in Japanese) (1994) The Journal of IEICE. 77 (8) 844852

(19) J. F, Abramatic \& L. M. Silverman: Nonlinear Restoration of Noisy Images (1982) IEEE Trans. Pattern Analysis and Machine Intelligence PAMI-4 (2) 141-149

(20) H. M. Kim \& B. Kosko: Fuzzy Prediction and Filtering in Impulsive Noise (1996) Fuzzy Sets and Systems 77 (1) 15-33

Noriaki Suetake (Non-member) received the B.E. and

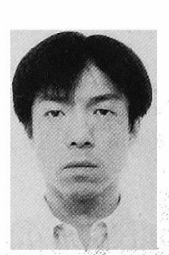
M.E. degrees in control engineering and science from the Kyushu Institute of Technology, Japan, in 1992 and 1994, respectively, and worked at Electronic \& Communication Systems Lab., Oki Electric Industry Co., Ltd. from 1994 to 1997 . He is presently a Ph.D. candidate at the Kyushu Institute of Technology, Iizuka, Japan. His current research interbio-inspired signal processing. ests include intelligent signal processing and

Takeshi Yamakawa (Non-member) received his Dr. Eng. degree in Electrochemistry from Tohoku University in 1974. He is presently a Professor of the Department of Control Engineering and Science, Kyushu Institute of Technology, Iizuka, Japan. He is also the Chairman of a Japanese foundation, Fuzzy Logic Systems Institute (FLSI), which was established by him for aiming at international collaboration on soft computing. He received Grigore Moisil Gold Medal in 1994 for his contribution to Engineering Application of Fuzzy Systems. He acted as Organizing/Program Committee members for over 60 international conferences and serves as associate editors of over 10 international journals in the field of soft computing. 